\title{
Nanobody nuclear imaging allows noninvasive quantification of LAG- 3 expression by tumor-infiltrating leukocytes and predicts response of immune checkpoint blockade
}

\section{RUNNING TITLE}

LAG-3 imaging predicts therapy response

Quentin Lecocq ${ }^{1}$, Robin Maximilian Awad ${ }^{1}$, Yannick De Vlaeminck ${ }^{1}$, Wout de Mey ${ }^{1}$, Thomas Ertveldt ${ }^{1}$, Cleo Goyvaerts ${ }^{1}$, Geert Raes ${ }^{2,3}$, Kris Thielemans ${ }^{1}$, Marleen Keyaerts ${ }^{4,5}$, Nick Devoogdt ${ }^{4}{ }^{*}$, Karine Breckpot ${ }^{1, *}$

${ }^{1}$ Laboratory for Molecular and Cellular Therapy, Vrije Universiteit Brussel, Laarbeeklaan 103, B-1090 Brussels, Belgium

${ }^{2}$ Myeloid Cell Immunology Lab, VIB Center for Inflammation Research, Brussels, Pleinlaan 2, B-1050 Brussels, Belgium

${ }^{3}$ Unit of Cellular and Molecular Immunology, Vrije Universiteit Brussel, Pleinlaan 2, B-1050 Brussels, Belgium

${ }^{4}$ In Vivo Cellular and Molecular Imaging Laboratory, Vrije Universiteit Brussel, Laarbeeklaan 103, B-1090 Brussels, Belgium

${ }^{5}$ Nuclear Medicine Department, UZ Brussel, Laarbeeklaan 101, B-1090 Brussels, Belgium

* These authors share senior authorship

Correspondence: Nick Devoogdt and Karine Breckpot

Email: nick.devoogdt@vub.be and karine.breckpot@vub.be

Contact first author: quentin.lecocq@vub.be

Immediate Open Access: Creative Commons Attribution 4.0 International License (CC BY) allows users to share and adapt with attribution, excluding materials credited to previous publications.

License: https://creativecommons.org/licenses/by/4.0/. Details: https://jnm.snmjournals.org/page/permissions.

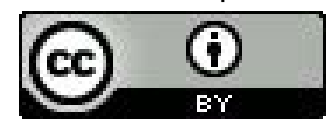




\section{ABSTRACT}

Recent advances in the field of immune-oncology led to the discovery of next-generation immune checkpoints (ICPs). Lymphocyte activation gene-3 (LAG-3), being the most widely studied amongst them, is being explored as a target for the treatment of cancer patients. Several antagonistic anti-LAG-3 antibodies are being developed and are prime candidates for clinical application. Furthermore, validated therapies targeting CTLA-4, PD-1 or PD-L1 showed that only subsets of patients respond. This finding highlights the need for better tools for patient selection and monitoring. The potential of molecular imaging to detect ICPs noninvasively in cancer is supported by several (pre)clinical studies. Here, we report on a nanobody to evaluate whole-body LAG-3 expression in various syngeneic mouse cancer models using nuclear imaging. The radiolabeled nanobody detected LAG-3 expression on tumor-infiltrating lymphocytes (TILs) as soon as 1 hour after injection in MC38, MO4 and TC-1 cancer models. The nanobody tracer visualized a compensatory upregulation of LAG-3 on TILs in MC38 tumors of mice treated with PD-1 blocking antibodies. When PD-1 blockade was combined with LAG-3 blockade, a synergistic effect on tumor growth delay was observed. These findings consolidate LAG-3 as a next-generation ICP and support the use of nanobodies as tools to noninvasively monitor the dynamic evolution of LAG-3 expression by TILs, which could be exploited to predict therapy outcome.

\section{KEYWORDS}

cancer; nuclear imaging; single domain antibody; nanobody; immune checkpoint; LAG-3 


\section{MAIN TEXT \\ INTRODUCTION}

A frequently exploited immunotherapy strategy in cancer is blockade of inhibitory immune checkpoints (ICPs) (1). So far, the FDA approved 7 antagonistic monoclonal antibodies (mAbs) against CTLA-4 (cytotoxic T-lymphocyte-associated protein-4), PD-1 (programmed cell-death protein-1) and its ligand PDL1 for treatment of cancer (2). Although groundbreaking and effective in subsets of patients, the response to CTLA-4 and PD-1/PD-L1 mAbs is not satisfactory, as most patients show primary or acquired resistance (3). This observation instigated research into novel ICPs, which could compensate for the loss of the targeted ICP. Of these, LAG-3 (lymphocyte activation gene-3) is a promising target with a high probability of clinical success $(4,5)$.

LAG-3 is a CD4-like molecule belonging to the immunoglobulin superfamily and is expressed on activated $\mathrm{CD}^{+}$and $\mathrm{CD} 8^{+}$T-cells (6), regulatory T-cells (7), B-cells (8), natural killer (NK)-cells (9), plasmacytoid dendritic cells (10) and myeloid cells like macrophages (11). Molecules such as MHC-II (12), Galectin-3 (13), LSECtin (14), a-synuclein (15) and fibrinogen-like protein-1 (16) can interact with LAG-3, with MHC-II being the canonical ligand. LAG-3 signaling is co-opted in the tumor microenvironment (TME) to enable tumor cell escape.

Relatlimab was the first anti-LAG-3 mAb that entered clinical testing, as a mono- or combination therapy with nivolumab, an anti-PD-1 mAb, in melanoma, renal cell carcinoma and non-small cell lung carcinoma (NSCLC) (NCT019680109). This phase-I trial showed that LAG-3 and PD-1-blockade is safe and restores T-cell functionality, leading to testing in phase-II trials. Today, 7 other mAbs and 3 antibodyderivatives that target LAG-3 are being evaluated in the clinic in a variety of malignancies. The high potential of LAG-3 blockade for cancer therapy is evidenced by the results in melanoma patients, who progressed despite previous non-LAG-3-related immunotherapy and who showed an $11 \%$ objective response rate (ORR) when treated with relatlimab, with one complete and 6 partial responders (17). In this study LAG-3 expression on nucleated cells within the tumor and invasive margin was determined using immunohistochemistry. It was observed that the ORR almost doubled in the LAG-3 expression group, underscoring the need for patient stratification.

Immunohistochemistry has several limitations with respect to ICP-detection and subsequent patient selection $(18,19)$. The need for a biopsy, the heterogeneous and dynamic expression of ICPs within the TME, and their role outside the TME are factors that can lead to incorrect stratification of patients. This could for instance explain why patients with undetectable PD-L1 levels showed beneficial effects of PDL1 blockade (20). As opposed to immunohistochemistry, nuclear imaging is a noninvasive process that can be performed repeatedly irrespective of the tumor location $(18,19,21)$. For example, imaging of PD-1 or PD-L1 using PET-tracers has been performed in clinical trials, showing a better correlation with therapy outcome than immunohistochemistry $(22,23)$. For sake of safety and clinical practicality, nuclear imaging should be fast and generate high-contrast images, which can be achieved with small, stable and soluble antigen-binding moieties. 
Nanobodies, the smallest antigen-binding fragment of camelid heavy-chain-only antibodies, are excellent tools to target proteins in the TME $(18,19)$. Nanobodies are small in size, easy to engineer and produce. Several radiolabeled nanobodies are currently evaluated in the clinic, including a Technetium99m ( ${ }^{99 m} \mathrm{TC}$ )-radiolabeled nanobody that targets PD-L1, showing specific uptake in patients with NSCLC at 2 hours after injection (24-27). Therefore, nanobodies targeting LAG-3 could be interesting diagnostic tools for noninvasive detection of LAG-3 before and after ICP-treatment.

We previously reported the characterization of nanobodies that target mouse LAG-3, showing that 99mTc-labeled nanobody 3132 is an excellent SPECT-probe to specifically detect LAG-3 on immune cells and on tumor cells that were engineered to express high levels of LAG-3 (28). In this study, we assessed the ability of this nanobody to image LAG-3 on tumor-infiltrating lymphocytes (TILs) in different mouse cancer models by SPECT/CT. We show that this nanobody can accurately quantify LAG-3 levels in the TIL-compartment. We moreover demonstrate the ability to detect LAG-3 upregulation on TILs in MC38 tumors of mice that were treated with anti-PD-1 mAbs. The enhanced therapy outcome in MC38-bearing mice treated with PD-1 and LAG-3-blocking mAbs corroborates the upregulation of LAG-3, as observed by nuclear imaging with the LAG-3 nanobody.

\section{MATERIALS AND METHODS}

\section{Mice, Cell lines \& Reagents}

Female, C57BL/6 mice (6-12 weeks) were purchased from Charles River (Ecully, France). The institution's ethical committee for use of laboratory animals approved the experiments. These were performed following the European guidelines for animal experimentation. MC38 mouse colorectal cancer cells and human embryonic kidney 293T cells were obtained from ATCC (Molsheim Cedex, France). MO4 melanoma cells were provided by K. Rock (University of Massachusetts Medical School, USA). These cells were cultured in Dulbecco's modified Eagle's medium supplemented with $10 \%$ fetal bovine serum (Tico Europe, Amstelveen, The Netherlands), 2mmol/l L-glutamine, 100U/ml penicillin and 100 $\mathrm{gg} / \mathrm{ml}$ streptomycin (PS). The TC-1 mouse lung cancer cells were provided by T.C. Wu (Johns Hopkins University, USA) and cultured in RPMI 1640 medium, supplemented with $10 \%$ fetal clone I serum (Thermo Fisher Scientific, Aalst, Belgium), L-glutamine, PS, $1 \mathrm{mmol} / /$ sodium pyruvate and non-essential amino acids, $12.5 \mathrm{mM} \mathrm{D}^{+}$-glucose, $5 \mathrm{mM}$ HEPES and $50 \mu \mathrm{M} \beta$-mercaptoethanol. Culture media and supplements were from Sigma-Aldrich (Zwijndrecht, Belgium) unless noted otherwise. A LAG-3-specific PerCPeFluor710 or PE-labeled antibody (Biolegend, clone eBioC9B7W) was used in flow cytometry. Antibodies from BD Biosciences were used to discriminate immune cells in flow cytometry: CD45.2-APC-Cy7 (clone 104), CD4-AF700 (clone RM4-5), CD8-V450 (clone 53-67), CD19-AF647 (clone 1D3), F4/80-BB700 (clone T452342).

\section{Nanobody production and quality control}


The LAG-3 nanobody 3132 was selected from a panel of candidates for its ability to bind to mouse LAG-3 (28). Nanobody R3B23, binding a multiple myeloma paraprotein (29), was used as a negative control. Nanobody production and quality control was performed as described (28).

\section{Inoculation of tumor cells and monitoring of tumor growth}

Mice were subcutaneously injected with $3 \times 10^{5}$ MC38, MO4 or TC-1 cells. Body weight, behavior, physical appearance and tumor ulceration were examined daily. Tumor dimensions were measured every other day using a caliper to calculate the tumor volume: (lengthxwidth $\left.{ }^{2}\right) / 2$. All procedures were performed under isoflurane anesthesia ( $5 \%$ for induction, $2.5 \%$ for maintenance with an oxygen flow of $1 \mathrm{~L} / \mathrm{min}$ ).

\section{ICP-blockade in tumor-bearing mice}

MC38-bearing mice were injected intraperitoneally with $10 \mathrm{mg} / \mathrm{kg}$ of Ultra-LEAF ${ }^{\mathrm{TM}} \mathrm{mAbs}$ (Biolegend, London, UK) targeting mouse PD-1 (clone RPM1-14), mouse LAG-3 (clone C9B7W), an isotype-matched control (IC) for the PD-1 monotherapy (rat IgG2a, clone RTK2758), or a mixture of ICmAbs for the anti-PD-1/LAG-3 combination therapy (rat lgG1, clone RTK2071 and rat IgG2a, clone RTK2758).

\section{Nanobody ${ }^{99 m}$ Tc-labeling, SPECT/CT-imaging, image and biodistribution analysis}

One hour before pinhole SPECT-micro-CT-imaging mice were intravenously injected with $5 \mu \mathrm{g}$ of 99mTc-labeled LAG-3 or control nanobody, with on average $68.8 \pm 6.8 \mathrm{MBq}$ and $88.9 \pm 5.1 \mathrm{MBq}$ of injected activity, resp. SPECT/CT-imaging was performed using a Vector ${ }^{+}$CT MiLABS scanner (MILabs, Utrecht, The Netherlands). SPECT-imaging was performed with a $1.5 \mathrm{~mm} 75$-pinhole general purpose collimator, in spiral mode with six bed positions. SPECT-scan time for total body was 15 minutes, 150 seconds per position. CT-scan time was 139 seconds in total, set to $60 \mathrm{kV}$ and $615 \mathrm{~mA}$. During all imaging procedures mice were anaesthetized by intraperitoneal injection with $75 \mathrm{mg} / \mathrm{kg}$ ketamine hydrochloride and $1 \mathrm{mg} / \mathrm{kg}$ medetomidine (Ketamidor, Richter Pharma AG, Austria). Immediately after imaging, organs of killed mice were isolated and weighed. Submandibular lymph nodes were selected as representative lymph nodes. The organ-specific uptake of each radiotracer was measured using a Wizard ${ }^{2} \gamma$-counter (PerkinElmer, Waltham, MA, USA). The uptake in each organ was corrected for decay and calculated as the percentage of injected activity per gram $(\% \mathrm{IA} / \mathrm{g})$. Image analysis was performed using AMIDE (Medical Image Data Examiner software) and HOROS medical imaging viewer (LGPL license at Horosproject.org). Isolated organs were kept in MACS $®$ tissue storage solution (Miltenyi Biotec, Leiden, Netherlands) when flow cytometry analysis was required.

\section{Single cell preparation of tumor and spleen}


Single cell suspensions of tumors and spleens were prepared according to protocols 130-096-730 and 130-095-926 of Miltenyi Biotec. Tumors were cut in pieces of approximately $3 \mathrm{~mm}$ and transferred to gentleMACS C tubes containing $5 \mathrm{ml}$ ice-cold RPMI1640 supplemented with $1000 \mathrm{U} / \mathrm{ml}$ DNAse I and 100 $\mu \mathrm{l}$ collagenase I. Tumors and whole spleens were homogenized at $37^{\circ} \mathrm{C}$ for respectively 45 and 15 minutes using the gentleMACS ${ }^{\top M}$ OCTO-dissociator (programs cus_37C_mlmpTu2 and 37C_m_SDK_1, respectively

). The cell suspension was filtered through a $70 \mu \mathrm{m}$ filter (BD Falcon), washed with phosphate buffered saline (PBS, Sigma-Aldrich) and centrifuged for 5 minutes at $1500 \mathrm{rpm}$. Red blood cells were removed by resuspending the cell pellet with $5 \mathrm{ml}$ lysis buffer $\left(0.16 \mathrm{M} \mathrm{NH}_{4} \mathrm{Cl}, 0.17 \mathrm{M}\right.$ Tris, $\left.\mathrm{pH} 7.2\right)$. After 2 minutes of incubation, $10 \mathrm{ml}$ PBS was added, cells were centrifuged, resuspended in cold PBS, counted (CasyTon, Innovatis) and prepared for flow cytometry staining and analysis.

\section{Flow cytometry}

Staining cell surface markers was previously described (30). Live/dead staining of the single cell preparations was performed using ZombieAqua ${ }^{\mathrm{TM}}$ (BV510, Biolegend). Blockade of Fc $\gamma$ Il and Fc $\gamma$ III receptors (CD16/32 antibody, clone 93, BioLegend) and staining of surface makers was performed in PBS containing $0.5 \% \mathrm{BSA}$ and $0.02 \% \mathrm{NaN}_{3}$. Cells were acquired on a FACSCelesta or LSRFortessa flow cytometer (BD Biosciences). Data were analyzed using FlowJo $X^{\circledR}$ software (Tree star, Ashland, Oregon, USA).

\section{Statistics}

Statistical analyses were performed with GraphPad Prism software (version 7.2). Data are represented as mean \pm standard deviation (SD). $p$-values were calculated using Mann-Whitney tests and Spearman correlation tests for flow cytometry and nanobody biodistribution experiments or log rank tests for survival experiments. The asterisks in the figures indicate statistical significance as: ${ }^{*} p<0.05$; ${ }^{* *} p<0.01$; ${ }^{* * *} p<0.001 ;{ }^{* * * *} p<0.0001$; n.s. not significant.

\section{RESULTS}

\section{Radiolabeled LAG-3 nanobodies allow imaging of LAG-3 in the TME}

We radiolabeled LAG-3 or control nanobodies with ${ }^{99 \mathrm{~m} T c}$ and compared their biodistribution in MC38-bearing mice by performing SPECT/CT at days 11 or 17 of tumor growth (Supplementary Fig. 1ac). The average injected dose and tumor size at the time of evaluation are shown in supplementary Figure 1d. Eighty minutes post-injection, we observed signals in kidneys and bladder, due to nanobody clearance, and for the LAG-3 nanobody tracer in tumors (Fig. 1). After imaging, organs were dissected, weighed and radioactivity levels measured (Fig. 2a). Analysis of tumor uptake showed that only radiolabeled LAG-3 nanobody accumulated in MC38-tumors with little increase in larger tumors ( $p=0.0364$ and $p=0.0091$ for 
day 11 and 17 tumors, resp.) (Fig. 2b). We assessed the accuracy of the radiolabeled nanobodies to image LAG-3 expression levels in the tumor by comparing ex vivo $\gamma$-counts with the activity that can be measured on SPECT/CT-scans using AMIDE-software (Fig. 2c). LAG-3 nanobody tumor uptake was also evaluated in mice bearing TC-1 or MO4 tumors. The average tumor size and the injected nanobody doses are shown in supplementary Figure 2a. Eighty minutes post-injection, LAG-3 nanobody accumulated in TC-1 and MO4 tumors (Supplementary Fig. 2b). Flow cytometry analysis showed LAG-3 expression on CD45 immune cells in TC-1 or MO4 tumors (Supplementary Fig. 2c,d). In both models, tracer uptake levels and mean fluoresscence intensity (MFI) of LAG3+ ${ }^{+}$TILs correlated positively (Supplementary Fig. 2d). Representative axial images of both tumor types are shown in supplementary Figure 2e.

\section{PD-1-blockade causes upregulation of LAG-3 on TILs}

We evaluated if we could detect LAG-3 upregulation on TILs during PD-1-blockade in the MC38model $(31,32)$. Tumor-bearing mice were treated with anti-PD-1 or control mAbs. SPECT/CT-imaging and ex vivo validations after injection of ${ }^{99 \mathrm{~m} T C}$-labeled LAG-3 nanobody was performed (Supplementary Fig. 3a).

We observed a slower tumor growth when mice were treated with PD-1-blocking mAbs compared to IC-mAbs (Supplementary Fig. 3b \& 4a; $p=0.0323$ ). We further analyzed SPECT/CT-images using quantification software (Fig. 3a) and calculated activity within the region of interest (ROI), assigned over the tumor mass. These values correlated positively with the $\gamma$-counts obtained through ex vivo analysis (Fig. 3b). Ex vivo biodistribution analysis showed a significantly higher uptake of LAG-3 nanobody in lymph nodes $(p=0.0037)$ and MC38-tumors $(p=0.0022)$, but not in spleen, as a result of PD-1-blockade (Supplementary Fig. 4b,c,d,e) (Supplementary Fig. 5a,b). LAG-3 nanobody tumor uptake in mice treated with anti-PD-1 or IC-mAbs was also compared with the MFI of LAG-3 expression on TILs, measured in flow cytometry. A positive correlation was found for CD45 total TILs, CD8+ T-cells, CD19+ B-cells and F4/80+ macrophages, but not for CD4 ${ }^{+}$T-cells (Fig. 4a). We also observed a significantly higher CD8 ${ }^{+} \mathrm{T}-$ cell infiltration in tumors of PD-1-treated mice relative to IC (Fig. $4 \mathrm{~b} ; p=0.0350$ ), but no differences in tumor infiltration for $\mathrm{CD}^{+}{ }^{+} \mathrm{T}$-cells, $\mathrm{CD} 19^{+} \mathrm{B}$-cells or $\mathrm{F} 4 / 80^{+}$macrophages (data not shown). Notably, we observed an increase of LAG-3 expression on total CD45+ TILs in mice treated with anti-PD-1 mAbs $(p=0.0221)$ (Fig. 4c). Although not significantly different between PD-1 and IC-treated groups, the proportion of LAG$3^{+} \mathrm{CD}^{+}$T-cells $(p=0.0518), \mathrm{CD} 19^{+} \mathrm{B}$-cells $(p=0.1807)$ and $\mathrm{F} 4 / 80^{+}$macrophages $(p=0.1807)$ tended to be more pronounced in mice treated with anti-PD-1 mAbs (Fig. 4d). Elevated expression of LAG-3 on TILs in mice treated with anti-PD-1 mAbs was further corroborated by an increase in MFI, which was statistically different from IC-treated group for CD19+ B-cells (Fig. 5, p=0.0140). Interestingly, no significant difference in LAG-3 expression was observed on spleen-residing immune cells of PD-1 and IC-treated groups (Fig. 5 ), which is in line with the spleen uptake values of radiolabeled LAG-3 nanobody (Supplementary Fig. 4d). 


\section{Blockade of LAG-3 in combination with PD-1-blockade enhances therapy outcome}

We next assessed whether blockade of LAG-3 and PD-1 in the MC38-model prolonged the tumor growth delay. Tumor-bearing mice were treated with anti-PD-1 and/or anti-LAG-3 mAbs as shown in Figure 6. Mice treated with a mixture of IC-mAbs served as a control. The delay in tumor growth upon treatment with anti-LAG-3 mAbs alone was not significant, while a statistical difference was observed between anti-PD-1 to IC mAb-treated mice (Fig. 6; $p=0.0201$ ). Combined PD-1/LAG-3-blockade further delayed tumor growth when compared to IC-mAbs $(p=0.0001)$, or monotherapy with anti-LAG-3 $(p=0.0201)$ or anti-PD-1 $(p=0.5257) \mathrm{mAbs}$. This translated in a significantly longer time to reach humane endpoints in mice receiving the combination therapy when compared to mice treated with IC $(p=0.0005)$, anti-LAG-3 $(p=0.0004)$ or anti-PD-1 $(p=0.0181)$ mAbs (Fig. 6$)$.

\section{DISCUSSION}

mAbs targeting ICPs like CTLA-4 and PD-1/PD-L1 have changed the field of immune-oncology due to their potent effects in a diversity of human cancers. However, there is still a fraction of patients that do not respond to this therapy. Additional ICPs, such as LAG-3, have been discovered, offering the potential to overcome resistance in some of these patients, by blocking LAG-3. Its important role in cancer development has been addressed in numerous pre-clinical studies $(28,32-37)$. It was shown that LAG-3 is expressed on T-cells, B-cells, plasmacytoid dendritic cells, NK-cells and macrophages $(1,6,11,28)$. Its induction is related to the dysfunction of cancer-specific T-cells often associated with PD-1 co-expression $(33,34,38)$. The latter could be an explanation of therapeutic resistance to single-agent ICP-blockade in patients. Subsequently, efforts have been made to develop anti-LAG-3 mAbs and explore their anticancer efficacy when used alone or in combination with anti-PD-1-blockade.

Evaluating the expression of ICPs within the tumor to select patients and predict their therapy outcome is of interest. Nuclear imaging using small binding moieties like nanobodies is a method to quickly detect biomarkers with high contrast in a noninvasive way $(18,21)$. Moreover, it can be performed repeatedly in the same patient regardless of the location of the tumor. Several clinical studies led to the observation that radiolabeled nanobodies are good candidates to detect tumor biomarkers with high contrast as soon as 60 minutes after administration to the patient $(19,26,27,39)$.

We were the first to report different nanobodies targeting mouse LAG-3 and to select one of these nanobodies for detection of LAG-3-engineered tumor cells (28). In this study, we evaluated this nanobody for detection of LAG-3-expressing TILs. We performed SPECT/CT-imaging in immunocompetent colon carcinoma-bearing mice using 99mTc-labeled LAG-3 nanobody and compared tumor uptake values to a radiolabeled control nanobody. Moreover, we performed imaging at days 11 or 17 of tumor growth to evaluate if tumor size alters LAG-3 detection with the nanobody tracer. Next to the specific signals detected in organs like the thymus, spleen and lymph nodes, we observed a significantly higher uptake of LAG-3 nanobody in tumors compared to control nanobody. No difference in nanobody uptake was observed when 
comparing weight-corrected uptake values in the tumors imaged at days 11 or 17 . The ex vivo measured tumor activities could be correlated to the SPECT/CT-images. We further extended our SPECT/CTimaging studies and ex vivo validations to a syngeneic melanoma and lung cancer model. The LAG3 nanobody tracer also accumulated in these tumors. Notably, uptake values correlated to the ex vivo evaluated LAG-3 expression on CD45+ TILs using flow cytometry. Overall, the SPECT/CT-images of these scans show the potency of the nanobody tracer to map LAG-3 in the TME.

LAG-3 expression can act as a compensatory mechanism that leads to therapeutic resistance of PD-1-blockade in cancer patients $(17,33,34,38)$. We are the first to explore the effects of PD-1-treatment on LAG-3 expression and distribution using molecular imaging. MC38-bearing mice were treated with PD1-blocking mAbs leading to tumor growth impairment. Noninvasive imaging revealed elevated LAG-3 nanobody tracer uptake in tumor and lymph nodes relative to the IC-treatment group. PD-1-treated mice had more $C D 8^{+}$T-cells in their tumor and more LAG-3+ TILs. A positive correlation was found between the amount of LAG-3 nanobody tracer in the tumor and the LAG-3 levels on CD8 ${ }^{+}$T-cells, CD19+ B-cells and $\mathrm{F} 4 / 80^{+}$macrophages. However, some individual samples were inconclusive when we attempted to correlate LAG-3 nanobody uptake levels with flow cytometry data. Intriguingly, previous studies suggest checkpoint blockade to cause changes in tumor environment like a reduction un tumor interstitial pressure and decompressed tumor blood vessels (40). Although proven for PD-L1 blocking antibodies, we must not exclude the possibility of similar anti-PD-1 mediated modifications that can alter the exposure of tumors to our radiolabeled LAG-3 nanobody tracer. Nevertheless, proper correlation of LAG-3 nanobody uptake levels with flow cytometry data could have been clarified by also analyzing tumors using immunohistochemistry as it would have allowed to more accurately quantify the percentage of immune cells per unit weight of tumor. Taken together, our findings support the idea of a compensatory upregulation of LAG-3 in the TME after PD-1-blockade. This compensatory LAG-3 expression in tumors upon PD-1-treatment could be reliably captured with high contrast with the LAG-3 nanobody SPECT/CTtracer.

As we observed a compensatory upregulation of LAG-3 in the TME after single-agent PD-1blockade, we evaluated the anticancer effects of combined LAG-3/PD-1-blockade in MC38-tumors. Tumor growth was marginally affected by LAG-3-blockade. However, when combined with PD-1-blockade, its anticancer effect surpasses the already significant decrease in tumor growth observed with PD-1blockade. This corroborates the compensatory role of LAG-3 upon PD-1-blockade in the MC38-model and underscores the benefit of combining blockade of multiple ICPs $(31,32)$.

As an increasing number of clinical trials investigate LAG-3-blockade in immunotherapy, most often in combination with PD-1-blockade, also a clinical tracer to monitor LAG-3 levels in the tumors of patients is of high interest. This study uses a nanobody binding mouse LAG-3 with no cross-reactivity with human LAG-3. We developed a nanobody that binds to human LAG-3 with low nanomolar affinity for human LAG-3 and a good capacity to visualize human LAG-3+ tumors in mice (unpublished data). Optimally this nanobody needs to be labeled with a short-lived PET-isotope such as ${ }^{68} \mathrm{Ga}$ or ${ }^{18} \mathrm{~F}$ using new 
or established radiochemistry procedures (25,41-43). Extrapolating from our still ongoing clinical trials with a ${ }^{68} \mathrm{Ga}$-labeled anti-HER2 nanobody breast cancer PET-tracer (26) and an anti-CD206 nanobody macrophage PET-tracer (25), we are hopeful that the future clinical anti-LAG-3 nanobody PET-tracer will also be safe, sensitive and will conveniently provide a whole-body picture of LAG-3 expression levels in a same-day imaging procedure with acceptable dosimetry levels.

\section{DECLARATIONS:}

\section{ACKNOWLEDGEMENTS}

The authors would like to thank Petra Roman and Elsy Vaeremans for the production of the plasmids and Cindy Peleman and Kevin de Jonghe for the labeling of the nanobodies and SPECT/CTimaging.

\section{DISCLOSURE STATEMENT}

Marleen Keyaerts received research funding from Precirix® (formerly named Camel-IDS). Quentin Lecocq, Karine Breckpot, Nick Devoogdt and Marleen Keyaerts have patents on the use of nanobodies for imaging and therapy. Nick Devoogdt and Marleen Keyaerts have ownership in AbScint, which leverages nanobody imaging tracers into clinical application. No other potential conflicts of interest relevant to this article exist.

\section{ABBREVIATIONS}

(CTLA-4) Cytotoxic T-lymphocyte-associated protein 4 (His6) Hexahistidine, (IC) Isotype control, (LAG-3) Lymphocyte-activation gene-3, (mAbs) Monoclonal antibodies, (MFI) Mean fluorescence intensity, (NK) Natural killer cells, (NSCLC) Non small cell lung cancer, (PD-1) Programmed cell-death protein-1, (PS) Penicillin streptomycin, (\%IA) Percentage injected activity, (ROI) Region of interest, (SD) Standard deviation, (99mTc) Technetium-99m.

\section{AUTHOR CONTRBUTIONS}

Conceptualization, Quentin Lecocq, Karine Breckpot, Nick Devoogdt, Marleen Keyaerts; Data curation, Quentin Lecocq, Maximillian Awad, Karine Breckpot, Nick Devoogdt, Marleen Keyaerts; Formal analysis, Quentin Lecocq, Wout De Mey, Thomas Ertveldt; Funding acquisition, Quentin Lecocq, Karine Breckpot, Nick Devoogdt, Marleen Keyaerts; Investigation, Quentin Lecocq, Maximillian Awad, Cleo Goyvaerts; Supervision, Geert Raes, Marleen Keyaerts, Karine Breckpot and Nick Devoogdt; Writing original draft, Quentin Lecocq; Writing - review \& editing, Quentin Lecocq, Maximillian Awad, Yannick De 
Vlaeminck, Wout De Mey, Thomas Ertveldt, Kris Thielemans, Geert Raes, Cleo Goyvaerts, Marleen Keyaerts, Karine Breckpot and Nick Devoogdt.

\section{FUNDING}

This research was financially supported by the Belgian Foundation against Cancer; Kom op tegen Kanker (Stand-up to Cancer), the Flemish cancer society; the Research Foundation Flanders (FWO-V; $1501019 N$ \& I001618N). Q. Lecocq has been funded via an "Emmanuel Vanderschueren" award and is a FWO-SB fellow (1S24218N).

\section{KEY POINTS}

QUESTION: Is detection of LAG-3 using radiolabeled nanobodies predictive for the therapeutic outcome of cancers treated with LAG-3-blocking moieties?

PERTINENT FINDINGS: This preclinical study describes the potential of a radiolabeled nanobody to noninvasively detect the compensatory upregulation of LAG-3 as a consequence of PD-1-blockade in cancer. The combination of PD-1 with LAG-3-blockade was able to significantly reduce tumor growth compared to monotherapy.

IMPLICATIONS FOR PATIENT CARE: Nanobodies, as tools to noninvasively monitor the dynamic evolution of LAG-3 expression by TILs, could be exploited to select patients and predict their therapy outcome. 


\section{REFERENCES}

1. Qin S, Xu L, Yi M, Yu S, Wu K, Luo S. Novel immune checkpoint targets: moving beyond PD-1 and CTLA-4. Mol Cancer. 2019;18:155.

2. Li Z, Song W, Rubinstein M, Liu D. Recent updates in cancer immunotherapy: a comprehensive review and perspective of the 2018 China Cancer Immunotherapy Workshop in Beijing. J Hematol Oncol. 2018;11:142.

3. O'Donnell JS, Long G V, Scolyer RA, Teng MWL, Smyth MJ. Resistance to PD1/PDL1 checkpoint inhibition. Cancer Treat Rev. 2017;52:71-81.

4. Andrews LP, Marciscano AE, Drake CG, Vignali DAA. LAG3 (CD223) as a cancer immunotherapy target. Immunol Rev. 2017;276:80-96.

5. Lecocq Q, Keyaerts M, Devoogdt N, Breckpot K. The Next-Generation Immune Checkpoint LAG3 and Its Therapeutic Potential in Oncology: Third Time's a Charm. Int J Mol Sci. 2021;22,75.

6. Triebel F, Jitsukawa S, Baixeras E, et al. LAG-3, a novel lymphocyte activation gene closely related to CD4. J Exp Med. 1990;171:1393-1405.

7. Huang C-T, Workman CJ, Flies D, et al. Role of LAG-3 in regulatory T cells. Immunity. 2004;21:503-513.

8. Kisielow M, Kisielow J, Capoferri-Sollami G, Karjalainen K. Expression of lymphocyte activation gene 3 (LAG-3) on B cells is induced by T cells. Eur J Immunol. 2005;35:2081-2088.

9. Huard B, Tournier M, Triebel F. LAG-3 does not define a specific mode of natural killing in human. Immunol Lett. 1998;61:109-112.

10. Andreae S, Piras F, Burdin N, Triebel F. Maturation and activation of dendritic cells induced by lymphocyte activation gene-3 (CD223). J Immunol. 2002;168:3874-3880.

11. Keane C, Law SC, Gould C, et al. LAG3: a novel immune checkpoint expressed by multiple lymphocyte subsets in diffuse large B-cell lymphoma. Blood Adv. 2020;4:1367-1377.

12. Baixeras E, Huard B, Miossec C, et al. Characterization of the lymphocyte activation gene 3- 
encoded protein. A new ligand for human leukocyte antigen class II antigens. J Exp Med. 1992;176:327-337.

13. Kouo T, Huang L, Pucsek AB, et al. Galectin-3 Shapes Antitumor Immune Responses by Suppressing CD8+ T Cells via LAG-3 and Inhibiting Expansion of Plasmacytoid Dendritic Cells. Cancer Immunol Res. 2015;3:412-423.

14. Xu F, Liu J, Liu D, et al. LSECtin expressed on melanoma cells promotes tumor progression by inhibiting antitumor T-cell responses. Cancer Res. 2014;74:3418-3428.

15. Mao X, Ou MT, Karuppagounder SS, et al. Pathological $\alpha$-synuclein transmission initiated by binding lymphocyte-activation gene 3. Science. 2016;353.

16. Wang J, Sanmamed MF, Datar I, et al. Fibrinogen-like Protein 1 Is a Major Immune Inhibitory Ligand of LAG-3. Cell. 2019;176:334-347.e12.

17. European Society for Medical Oncology. ESMO 2017 Congress Scientific Meeting Report. https://oncologypro.esmo.org/content/download/126251/2385263/file/ESMO-2017-CongressScientific-Meeting-Report.pdf .Accessed December 8, 2020.

18. Broos K, Lecocq Q, Raes G, Devoogdt N, Keyaerts M, Breckpot K. Noninvasive imaging of the PD-1:PD-L1 immune checkpoint: Embracing nuclear medicine for the benefit of personalized immunotherapy. Theranostics. 2018;8:3559-3570.

19. Lecocq Q, De Vlaeminck $Y$, Hanssens $\mathrm{H}$, et al. Theranostics in immuno-oncology using nanobody derivatives. Theranostics. 2019;9:7772-7791.

20. Teng MWL, Ngiow SF, Ribas A, Smyth MJ. Classifying Cancers Based on T-cell Infiltration and PD-L1. Cancer Res. 2015;75:2139-2145.

21. Du Y, Jin $Y$, Sun $W$, Fang J, Zheng J, Tian J. Advances in molecular imaging of immune checkpoint targets in malignancies: current and future prospect. Eur Radiol. 2019;29:4294-4302.

22. Bensch F, van der Veen EL, Lub-de Hooge MN, et al. (89)Zr-atezolizumab imaging as a noninvasive approach to assess clinical response to PD-L1 blockade in cancer. Nat Med. 
2018;24:1852-1858.

23. Niemeijer AN, Leung D, Huisman MC, et al. Whole body PD-1 and PD-L1 positron emission tomography in patients with non-small-cell lung cancer. Nat Commun. 2018;9:4664.

24. Broisat A, Toczek J, Dumas LS, et al. 99mTc-CAbVCAM1-5 imaging is a sensitive and reproducible tool for the detection of inflamed atherosclerotic lesions in mice. $J$ Nucl Med. 2014;55:1678-1684.

25. Xavier C, Blykers A, Laoui D, et al. Clinical Translation of [(68)Ga]Ga-NOTA-anti-MMR-sdAb for PET/CT Imaging of Protumorigenic Macrophages. Mol imaging Biol MIB Off Publ Acad Mol Imaging. January 2019;21:898-906.

26. Keyaerts M, Xavier C, Heemskerk J, et al. Phase I Study of 68Ga-HER2-Nanobody for PET/CT Assessment of HER2 Expression in Breast Carcinoma. J Nucl Med. 2016;57:27-33.

27. Xing Y, Chand G, Liu C, et al. Early Phase I Study of a (99m)Tc-Labeled Anti-Programmed Death Ligand-1 (PD-L1) Single-Domain Antibody in SPECT/CT Assessment of PD-L1 Expression in Non-Small Cell Lung Cancer. J Nucl Med. 2019;60:1213-1220.

28. Lecocq Q, Zeven K, De Vlaeminck Y, et al. Noninvasive Imaging of the Immune Checkpoint LAG3 Using Nanobodies, from Development to Pre-Clinical Use. Biomolecules. 2019;9:548.

29. Lemaire M, D'Huyvetter M, Lahoutte $T$, et al. Imaging and radioimmunotherapy of multiple myeloma with anti-idiotypic Nanobodies. Leukemia. 2014;28:444-447.

30. Breckpot K, Dullaers M, Bonehill A, et al. Lentivirally transduced dendritic cells as a tool for cancer immunotherapy. J Gene Med. 2003;5:654-667.

31. Yu X, Huang X, Chen X, et al. Characterization of a novel anti-human lymphocyte activation gene 3 (LAG-3) antibody for cancer immunotherapy. MAbs. June 2019:1-10.

32. Burova E, Hermann A, Dai J, et al. Preclinical Development of the Anti-LAG-3 Antibody REGN3767: Characterization and Activity in Combination with the Anti-PD-1 Antibody Cemiplimab in Human PD-1xLAG-3-Knockin Mice. Mol Cancer Ther. 2019;18:2051-2062. 
33. Huang R-Y, Francois A, McGray AR, Miliotto A, Odunsi K. Compensatory upregulation of PD-1, LAG-3, and CTLA-4 limits the efficacy of single-agent checkpoint blockade in metastatic ovarian cancer. Oncoimmunology. 2017;6:e1249561.

34. Huang R-Y, Eppolito C, Lele S, Shrikant P, Matsuzaki J, Odunsi K. LAG3 and PD1 co-inhibitory molecules collaborate to limit CD8+ T cell signaling and dampen antitumor immunity in a murine ovarian cancer model. Oncotarget. 2015;6:27359-27377.

35. Woo S-R, Turnis ME, Goldberg M V, et al. Immune inhibitory molecules LAG-3 and PD-1 synergistically regulate T-cell function to promote tumoral immune escape. Cancer Res. 2012;72:917-927.

36. Harris-Bookman S, Mathios D, Martin AM, et al. Expression of LAG-3 and efficacy of combination treatment with anti-LAG-3 and anti-PD-1 monoclonal antibodies in glioblastoma. Int $\mathrm{J}$ cancer. 2018;143:3201-3208.

37. Matsuzaki J, Gnjatic S, Mhawech-Fauceglia P, et al. Tumor-infiltrating NY-ESO-1-specific CD8+ T cells are negatively regulated by LAG-3 and PD-1 in human ovarian cancer. Proc Natl Acad Sci U S A. 2010;107:7875-7880.

38. Nagasaki J, Togashi $\mathrm{Y}$, Sugawara $\mathrm{T}$, et al. The critical role of CD4+ T cells in PD-1 blockade against MHC-II-expressing tumors such as classic Hodgkin lymphoma. Blood Adv. 2020;4:40694082.

39. Rashidian M, Ploegh H. Nanobodies as non-invasive imaging tools. IOTECH. 2020;7:2-14.

40. Clift R, Souratha J, Garrovillo SA, Zimmerman S, Blouw B. Remodeling the Tumor Microenvironment Sensitizes Breast Tumors to Anti-Programmed Death-Ligand 1 Immunotherapy. Cancer Res. 2019;79:4149-4159.

41. Bridoux J, Broos K, Lecocq Q, et al. Anti-human PD-L1 Nanobody for Immuno-PET Imaging: Validation of a Conjugation Strategy for Clinical Translation. Biomolecules. 2020;10,1388.

42. Cleeren F, Lecina J, Ahamed M, et al. Al(18)F-Labeling Of Heat-Sensitive Biomolecules for 
Positron Emission Tomography Imaging. Theranostics. 2017;7:2924-2939.

43. Blykers A, Schoonooghe S, Xavier C, et al. PET Imaging of Macrophage Mannose ReceptorExpressing Macrophages in Tumor Stroma Using 18F-Radiolabeled Camelid Single-Domain Antibody Fragments. J Nucl Med. 2015;56:1265-1271. 


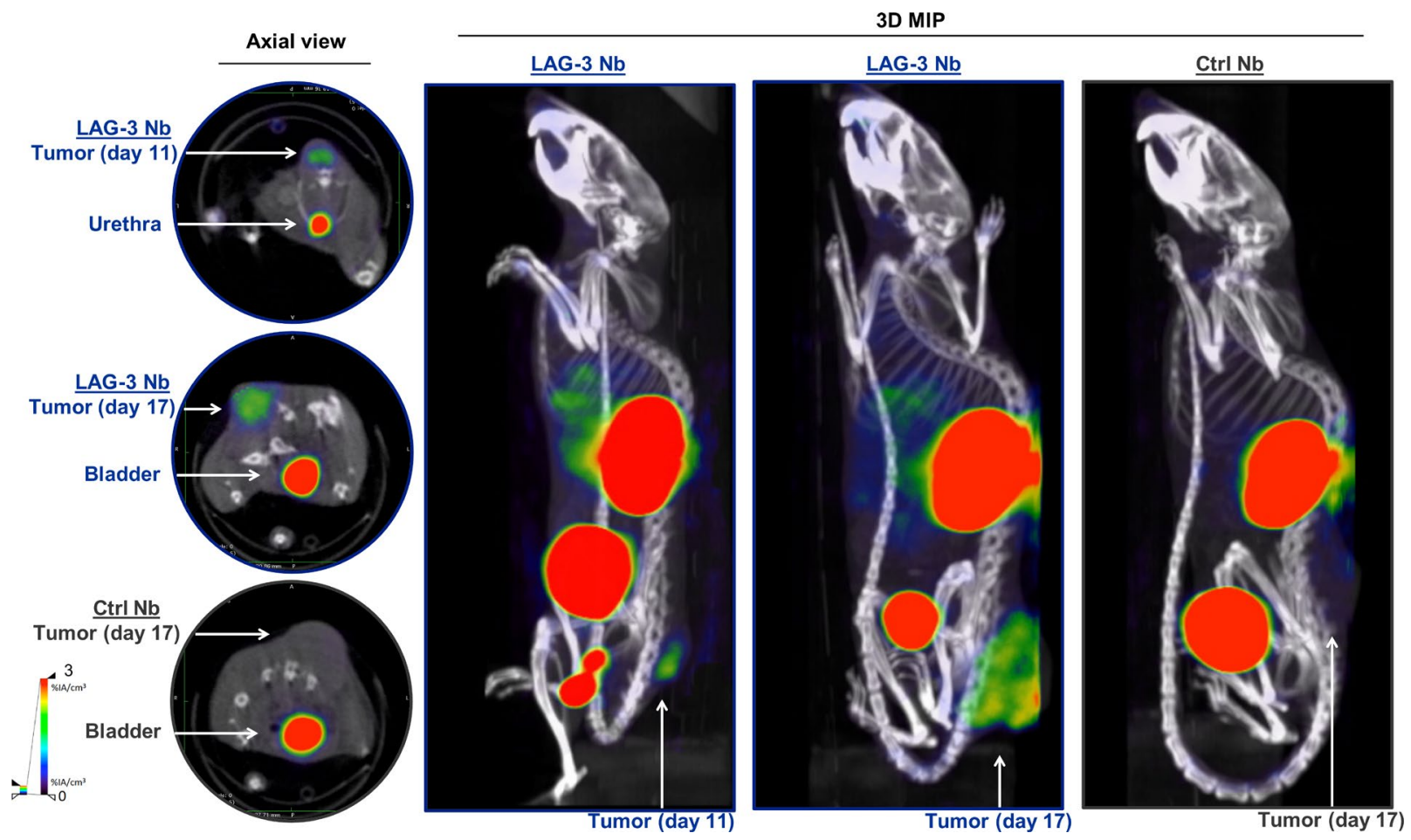

Figure 1: SPECT/CT-imaging of LAG-3 using ${ }^{99 m T C-l a b e l e d ~ L A G-3 ~ n a n o b o d y ~ i n ~ M C 38-b e a r i n g ~ m i c e ~ a t ~}$ days 11 or 17 of tumor growth. The figure shows representative SPECT/CT-images of MC38-bearing mice i.v. injected with ${ }^{99 m}$ Tc-labeled LAG-3 or control nanobodies. 

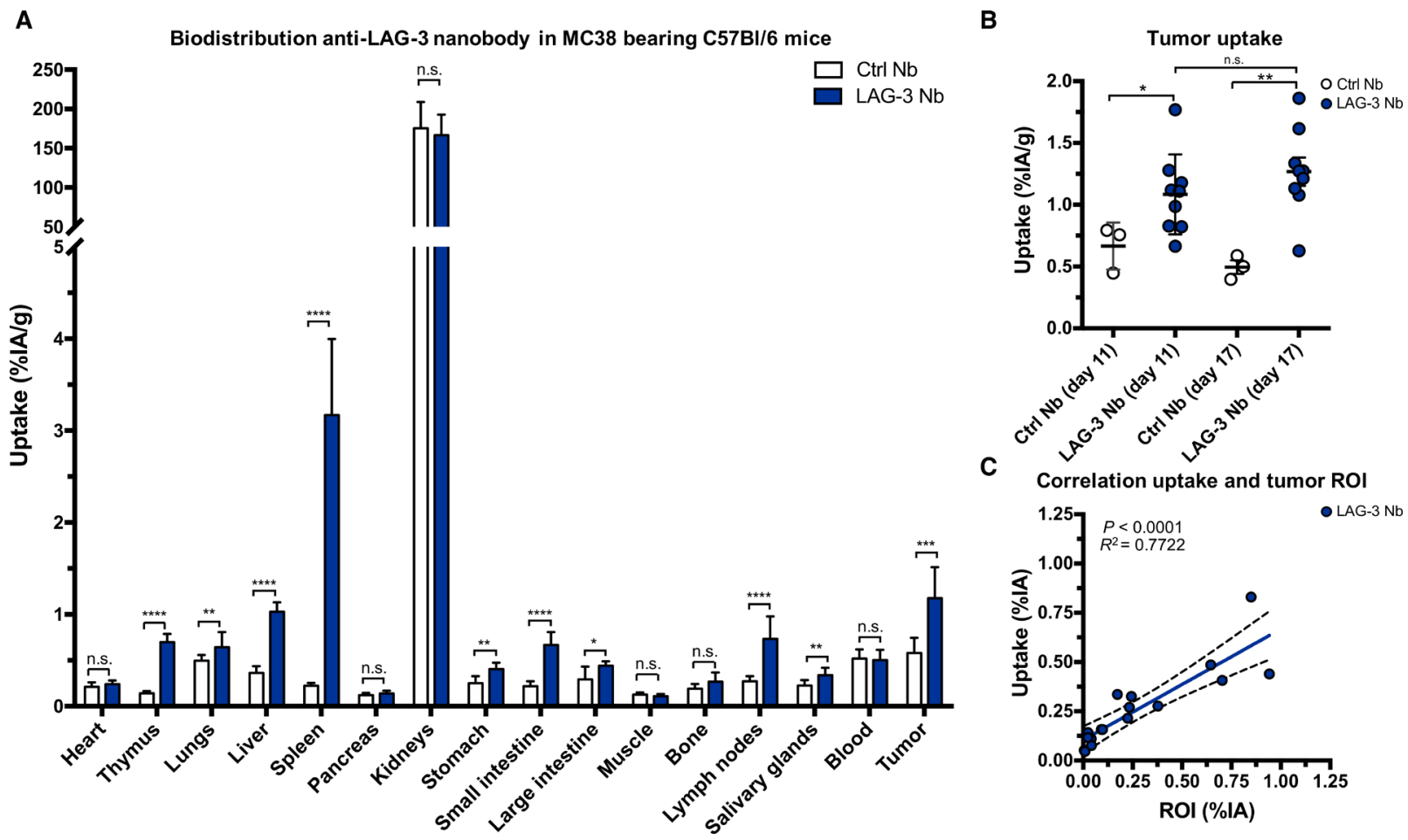

Figure 2: Biodistribution of radiolabeled LAG-3 and control nanobodies in MC38-bearing mice at days 11 or 17 of tumor growth. (a) Ex vivo $\gamma$-counting of isolated organs from MC38-bearing mice 80 minutes after injection of LAG-3 or control nanobody tracers. (b) Individual tumor uptake levels of LAG-3 and control nanobody tracers as determined by ex vivo $\gamma$-counting. (c) Correlation plot of tumor uptake within the image ROI (x-axis) and the ex vivo $\gamma$-counting of the tumors (y-axis) for the LAG-3 nanobody tracer. 

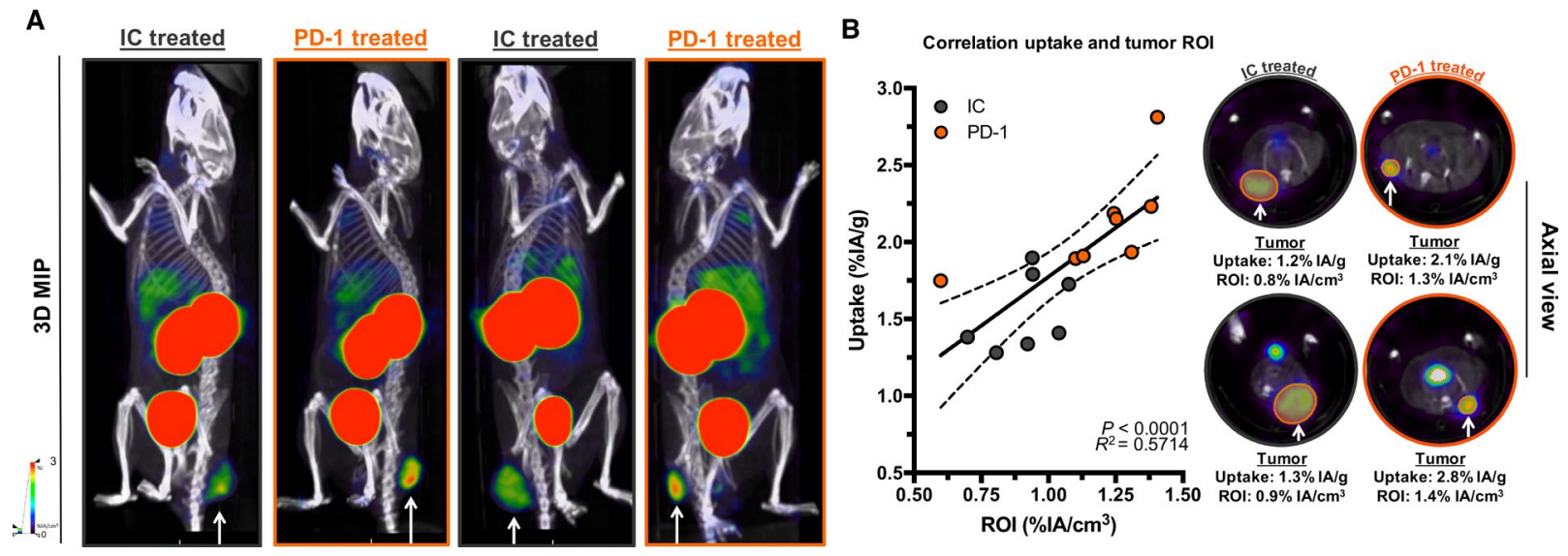

Figure 3: Effect of PD-1-blockade versus IC in MC38-bearing mice on LAG-3 detection using 99mTCnanobody SPECT/CT-imaging. (a) Representative SPECT/CT-images of mice treated with IC versus PD1-blocking mAbs. (b) Correlation plot and representative axial scans showing tumor signals in ROls (in $\% \mathrm{IA} / \mathrm{cm}^{3}, \mathrm{x}$-axis) and ex vivo $\gamma$-counting of tumors from IC and PD-1-treated mice (in \% IA/g, y-axis). 
A
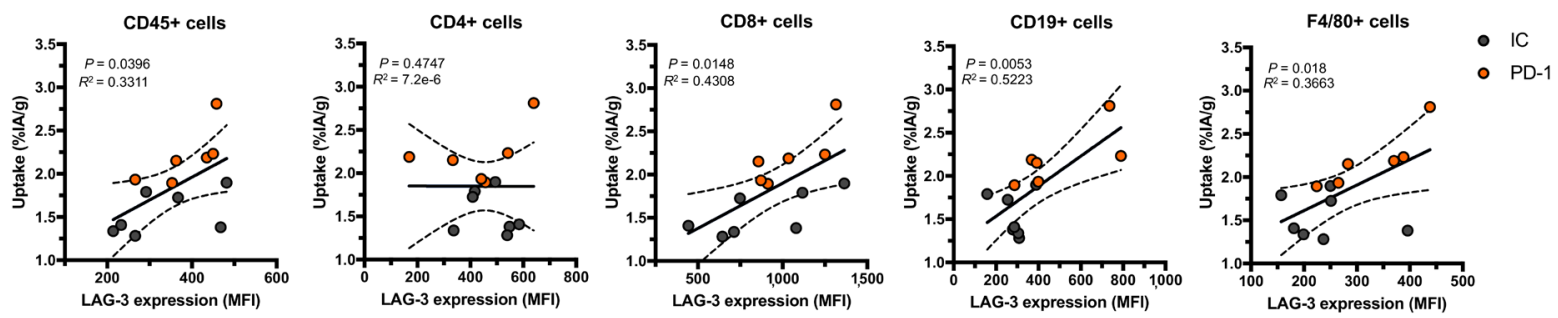

B

CD8 infiltration (\% of CD45+)
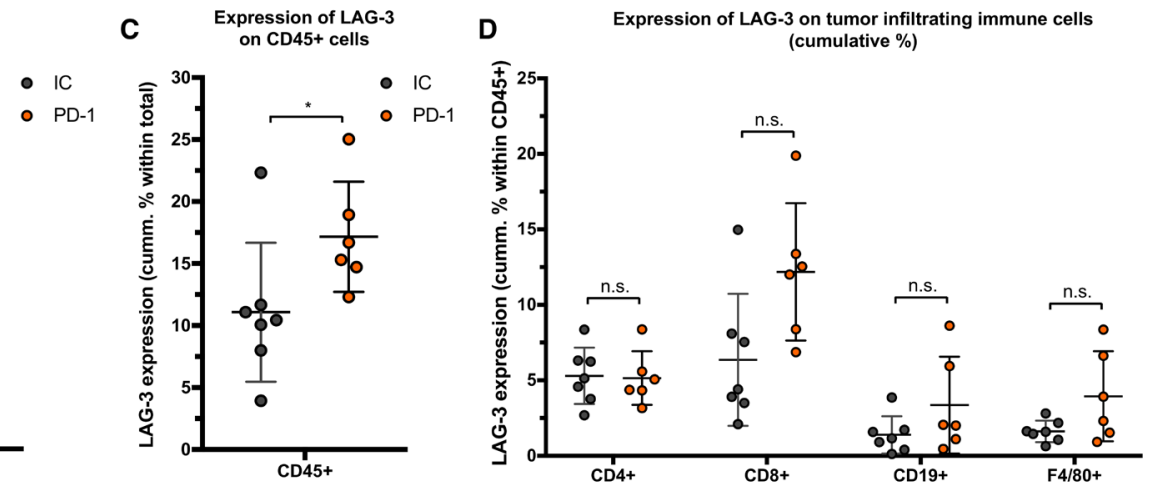

Figure 4: Ex vivo analysis of LAG-3 expression on immune cell populations within MC38-tumors of antiPD-1 or IC-treated mice. (a) Correlation plot showing LAG-3 expression (MFI) on different immune cell subsets as analyzed by flow cytometry (x-axis) and ex vivo $\gamma$-counting results of tumors (\%/A/g, y-axis). (b) Frequency of CD8+ T-cells in MC38-tumors as analyzed by flow cytometry. (c-d) Expression of LAG-3 (cumulative \%) on different immune cell subsets in MC38-tumors of anti-PD-1 ( $n=6)$ or IC-treated mice $(n=7)$ as analyzed by flow cytometry. 

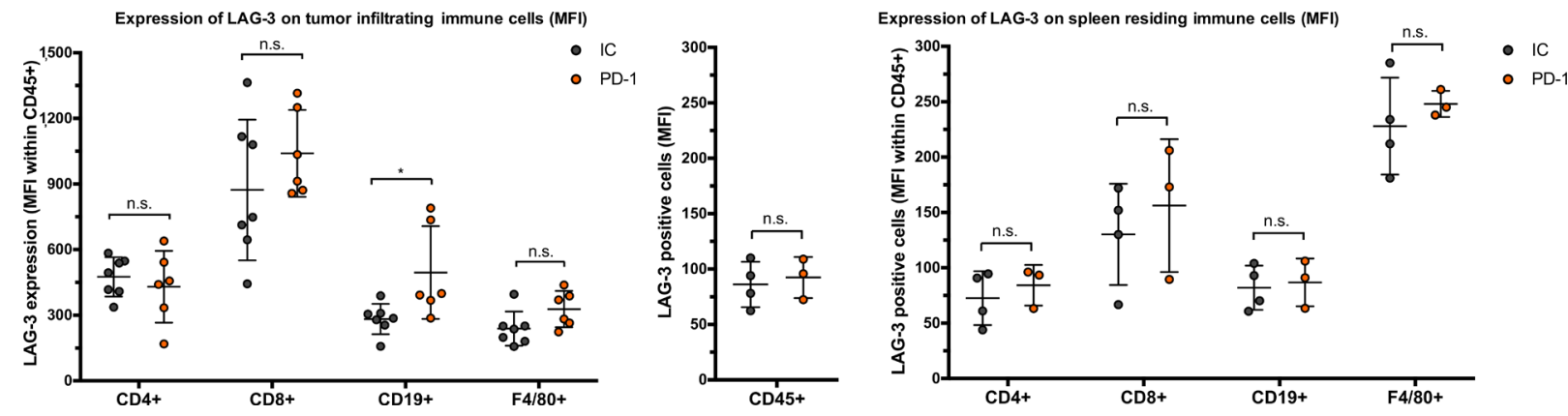

Figure 5: Ex vivo analysis of LAG-3 expression on immune cell populations within the tumor or spleen of anti-PD-1 or IC-treated mice. The left graph illustrates the expression of LAG-3 (MFI) on different immune cell subsets in MC38-tumors of anti-PD-1 $(n=6)$ or IC-treated $(n=7)$ mice, as analyzed by flow cytometry. The middle and right graphs illustrate the expression of LAG-3 (MFI) on different immune cell subsets in spleens of anti-PD-1 $(n=3)$ or IC-treated $(n=4)$ mice, as analyzed by flow cytometry. 

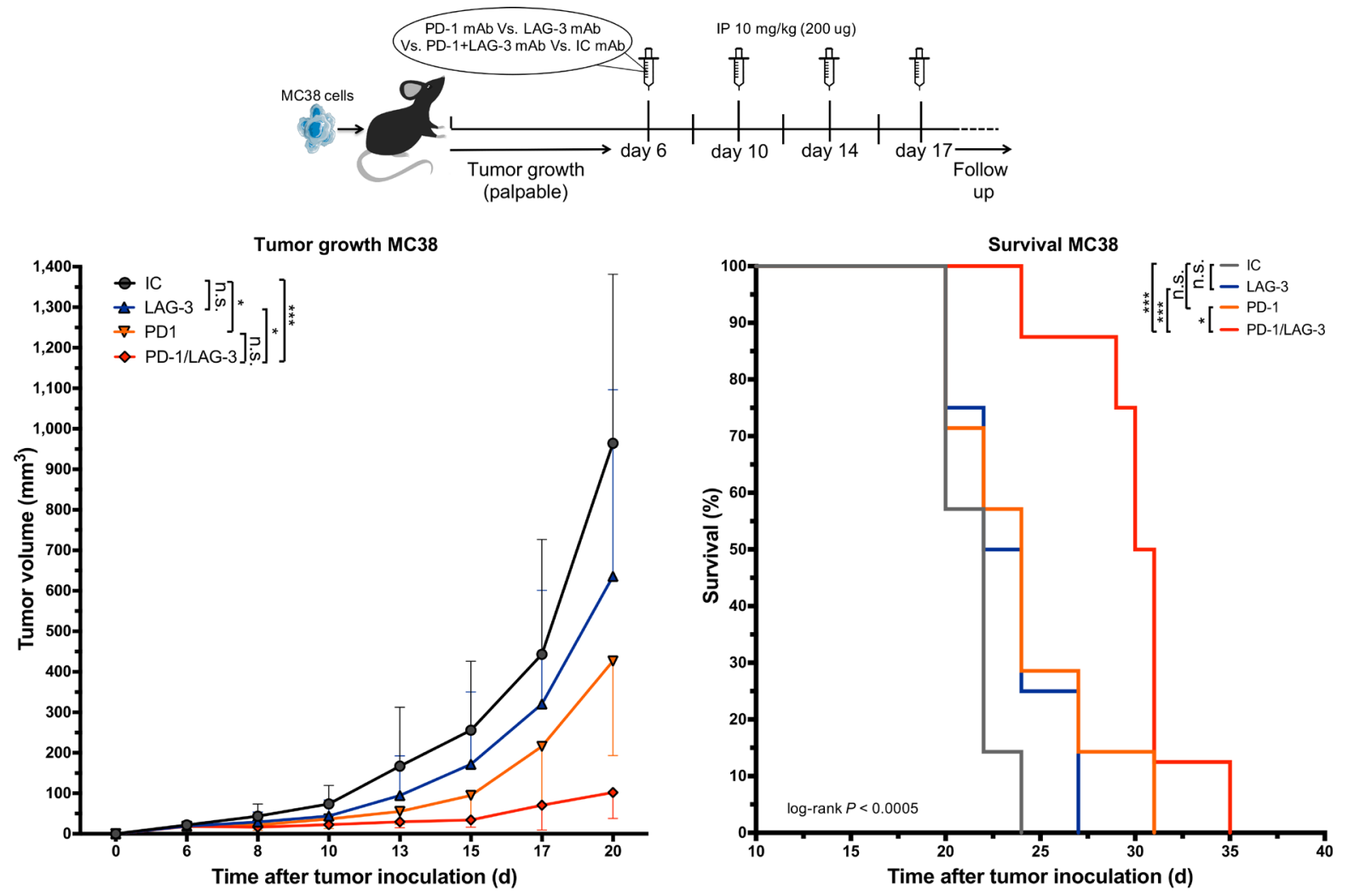

Figure 6: Response of established MC38-tumors to PD-1 and/or LAG-3-blockade. The top of the figure illustrates the timeline of the experiment. The left curve displays the growth kinetics of MC38-tumors treated with mAbs targeting PD-1 $(n=8)$, LAG-3 $(n=8)$, the combination of PD-1 and LAG-3 $(n=8)$, or the corresponding IC mAbs $(n=8)$. On the right, Kaplan-Meyer survival curves are shown (time to reach humane endpoints, $1500 \mathrm{~mm}^{3}$ tumor volume) 


\section{Graphical Abstract}

\section{Detection of LAG-3 on TILs upon anti-PD-1 therapy}

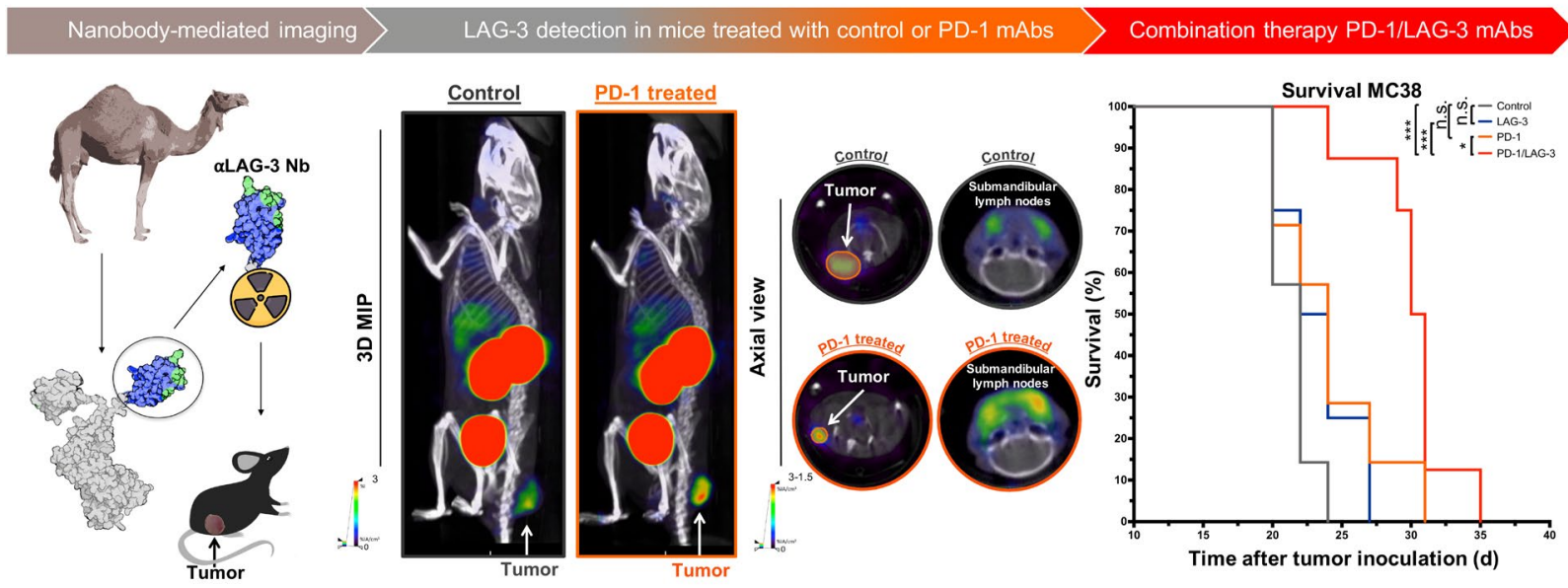




\section{Supplementary figures}

A

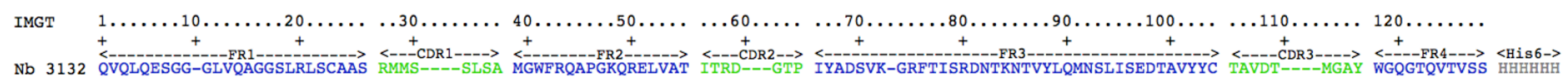

B

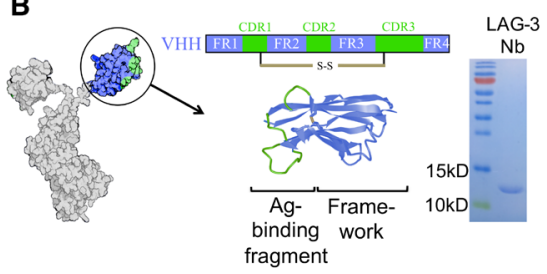

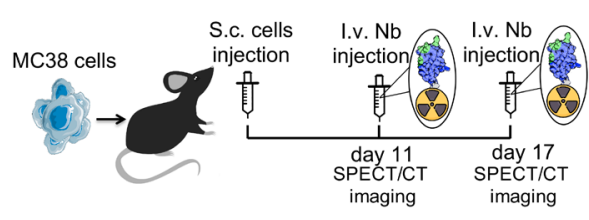

\begin{tabular}{|c||c|c|c}
\hline Nb & $\begin{array}{c}\text { Dose at } \\
\text { injection } \\
\text { (MBq) }\end{array}$ & $\begin{array}{c}\text { Tumor } \\
\text { size day } \\
11\left(\mathrm{~mm}^{3}\right)\end{array}$ & $\begin{array}{c}\text { Tumor } \\
\text { size day } \\
17\left(\mathrm{~mm}^{3}\right)\end{array}$ \\
\cline { 2 - 5 } Ctrl Nb & $\begin{array}{c}88.9 \pm \\
5.1\end{array}$ & $\begin{array}{c}106.3 \pm \\
113.1\end{array}$ & $\begin{array}{c}166.5 \pm \\
113.9\end{array}$ \\
\hline \multirow{2}{*}{ LAG-3 Nb } & $\begin{array}{c}65.5 \pm \\
5.6\end{array}$ & $\begin{array}{c}74.2 \pm \\
33.4\end{array}$ & $\begin{array}{c}315.6 \pm \\
220.2\end{array}$ \\
\hline
\end{tabular}

Supplementary Figure 1: Injection of 99mTC-labeled LAG-3 nanobody "3132" in MC38-bearing mice. (a) Amino acid sequence of the nanobody. (b) Structural model of a llama light-chain-deficient antibody from which the nanobody is derived and a Coomassie blue-stained protein gel loaded with $20 \mu \mathrm{g}$ of purified LAG-3 nanobody. (c) Timeline of the experiment. (d) Dose of injected radiolabeled LAG-3 nanobody $(\mathrm{n}=$ 18) or control nanobodies $(n=6)$ in MC38-bearing mice and the average tumor size at day of imaging. 
Uptake LAG-3 nanobody in tumor

\begin{tabular}{|c||c|c|}
\hline $\begin{array}{c}\text { Tumor } \\
\text { model }\end{array}$ & $\begin{array}{c}\text { Dose at } \\
\text { injection } \\
\text { (MBq) }\end{array}$ & $\begin{array}{c}\text { Tumor size at } \\
\text { imaging } \\
\left(\mathrm{mm}^{3}\right)\end{array}$ \\
\hline TC-1 & $77.5 \pm 4.8$ & $722 \pm 73$ \\
\hline MO4 & $67.4 \pm 4.6$ & $694 \pm 164$ \\
\hline
\end{tabular}

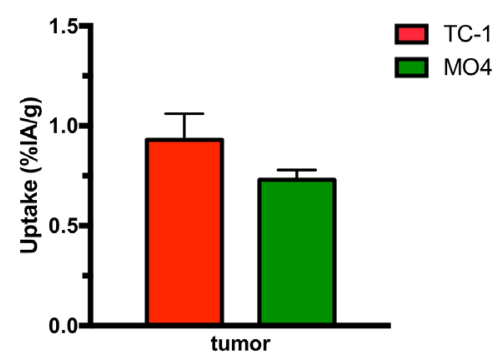

C

LAG-3 expression on CD45 cells

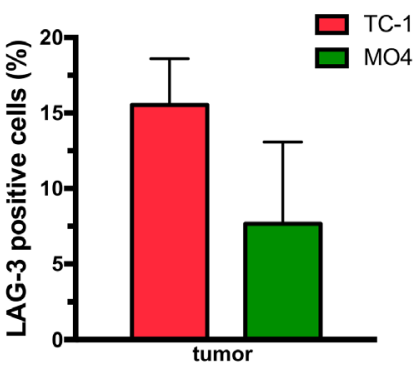

D

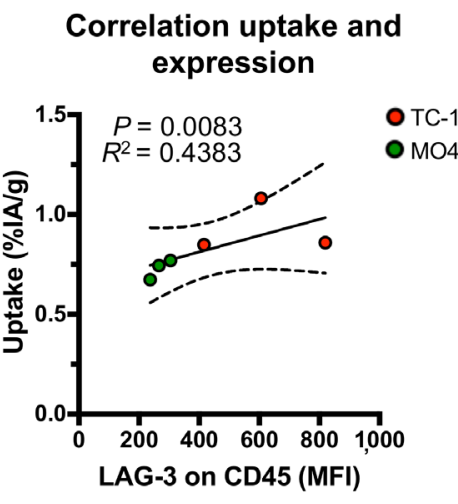

E

E $\quad$ TC-1 tumor

MO4 tumor
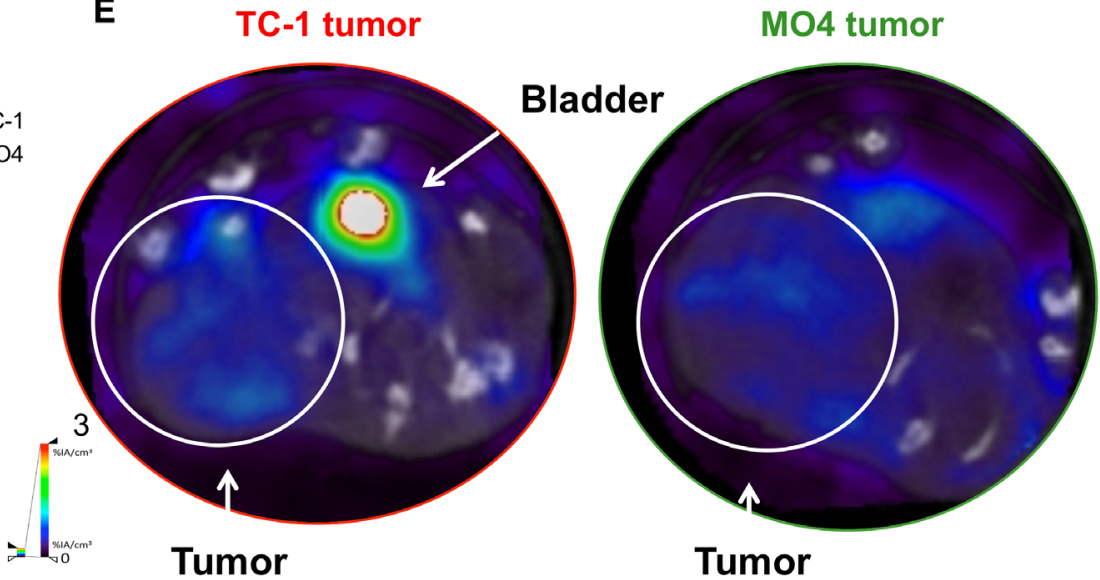

Supplementary Figure 2: SPECT/CT-imaging of LAG-3 using radiolabeled LAG-3 nanobody in TC-1 or MO4-bearing mice. (a) Dose of injected radiolabeled LAG-3 nanobody in mice bearing TC-1 $(n=3)$ or MO4 $(n=3)$ tumors and tumor volume at the time of imaging. (b) LAG-3 nanobody tracer tumor uptake levels as determined by ex vivo $\gamma$-counting of dissected tumors. (c) Percentage of LAG- $3^{+} / \mathrm{CD} 45^{+}$immune cells in tumors. (d) Correlation plot of LAG-3 expression (MFI) on immune cells (CD45+) in TC-1 or MO4 tumors as analyzed by flow cytometry (x-axis) and the ex vivo $\gamma$-counting of the tumors (\%/A/g, $y$-axis). (e) Representative axial SPECT/CT-images of TC-1 or MO4-bearing mice i.v. injected with ${ }^{99 \mathrm{~m} T \mathrm{~T}-\text {-labeled }}$ LAG-3 nanobody. 
A
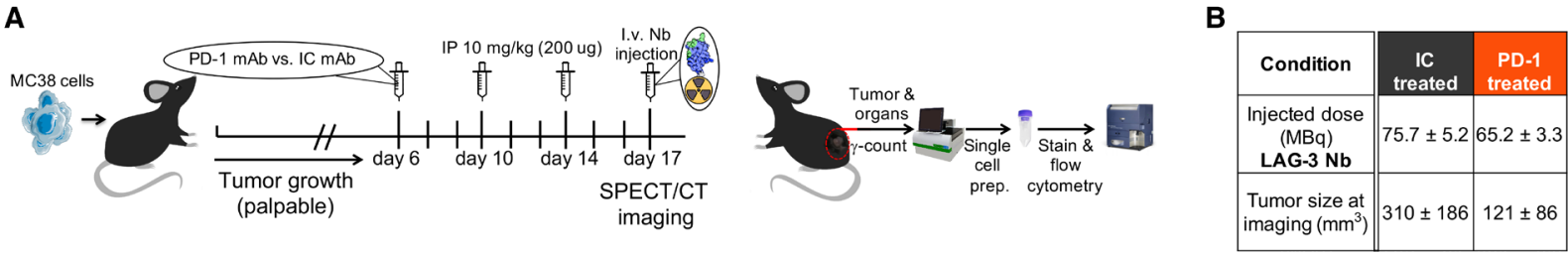

Supplementary Figure 3: Evaluating PD-1-blockade versus IC in MC38-bearing mice. (a) Timeline of the experiment. (b) Tumor size at time of imaging and dose of injected radiolabeled LAG-3 nanobody in MC38bearing mice treated with either IC-mAbs $(n=7)$ or PD-1-blocking mAbs $(n=8)$. 


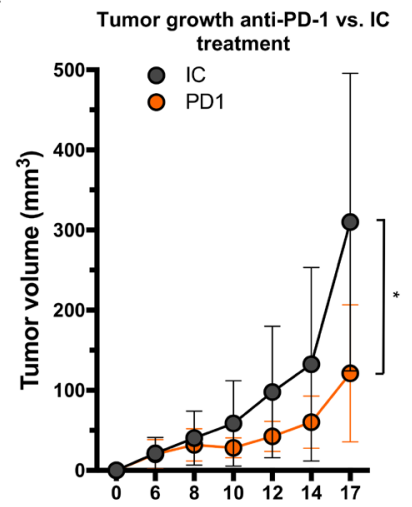

Time after tumor inoculation (d)
B

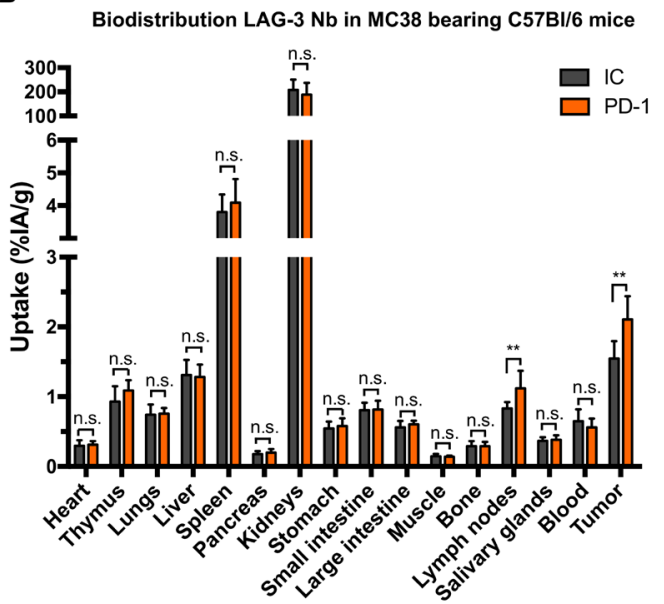

$\mathbf{E}$

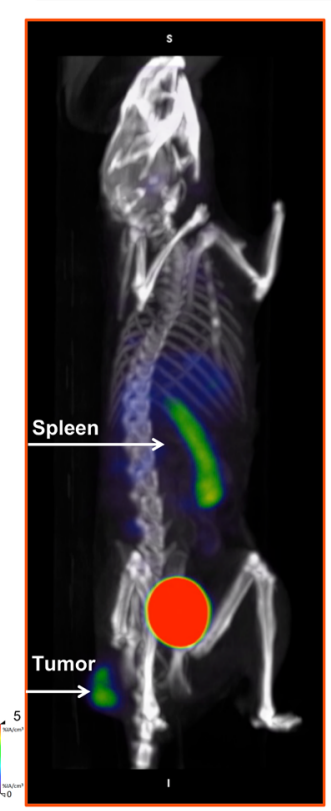

3D MIP

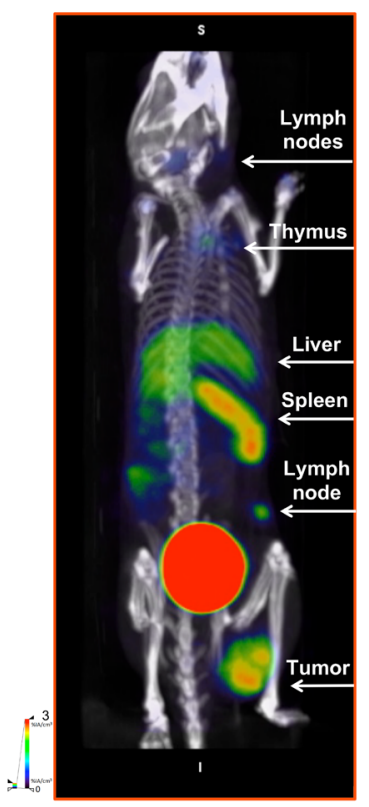

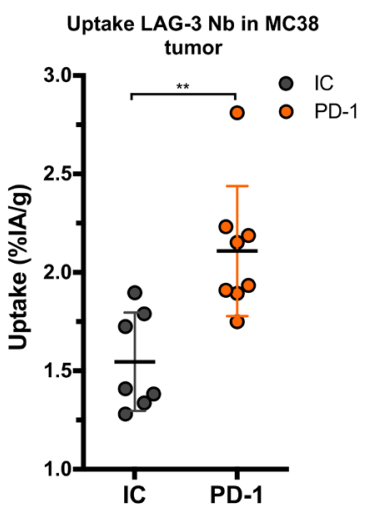

D

Uptake LAG-3 $\mathrm{Nb}$ in spleen

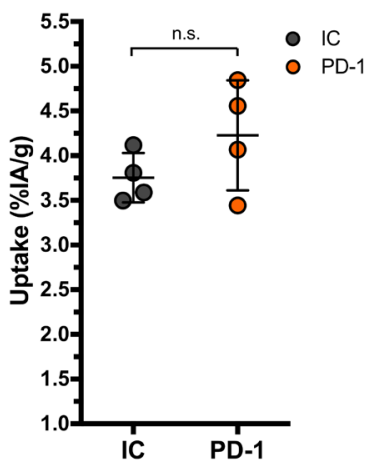

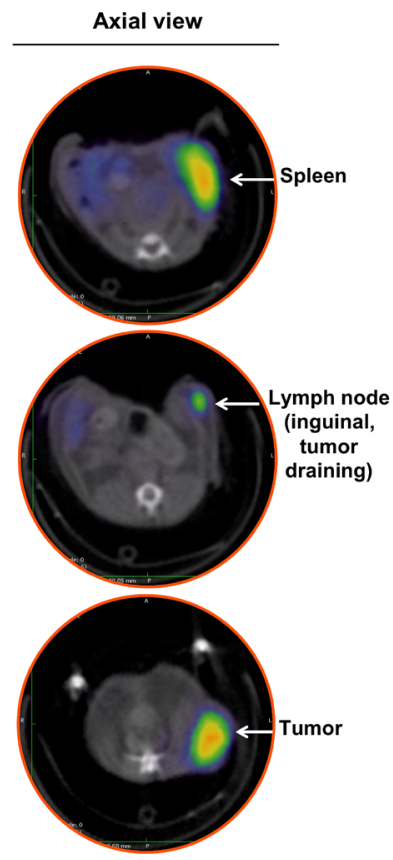

Supplementary Figure 4: Effect of PD-1-blockade versus IC in MC38-bearing mice on LAG-3 detection in the tumor and spleen. (a) Growth kinetics of MC38-tumors treated with IC-mAbs ( $n=7)$ or PD-1-blocking mAbs (n=8). (b) Ex vivo $\gamma$-counting of isolated organs from MC38-bearing mice, treated with anti-PD-1 or IC, 80 minutes after injection of LAG-3 nanobody tracer. (c) Uptake levels of LAG-3 nanobody tracer in MC38-tumors of anti-PD-1 or IC-treated mice, as determined by ex vivo $\gamma$-counting. (d) Uptake levels of LAG-3 nanobody tracer in spleens of anti-PD-1 or IC-treated mice, as determined by ex vivo $\gamma$-counting. (e) SPECT/CT-imaging of anti-PD-1 treated mice i.v. injected with LAG-3 nanobody tracer, scaled at 0-5 (left) or 0-3\% $\mathrm{IA} / \mathrm{cm}^{3}$ (right), showing specific signals in the spleen, tumor and tumor-draining lymph node $(n=2)$. Note the removal of both kidneys before image acquisition. 
A

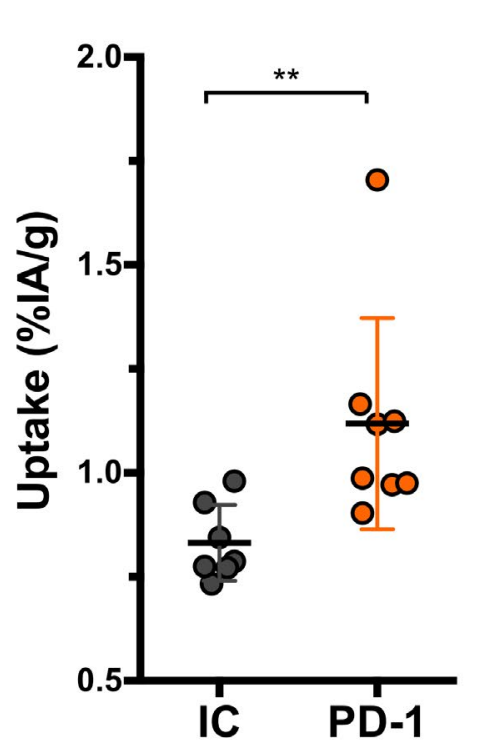

- IC lymph nodes (IC

- PD-1 treated)
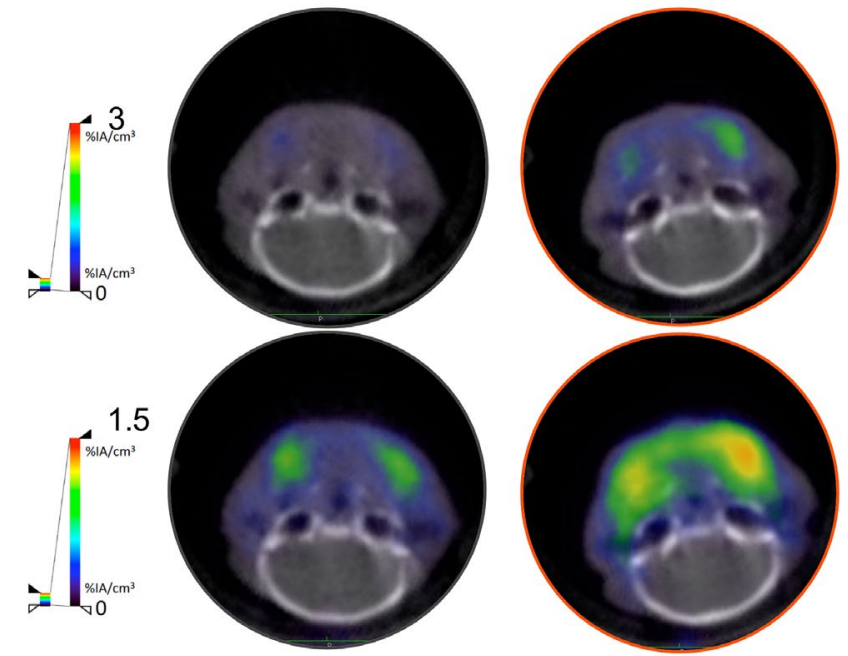

Supplementary Figure 5: Effect of PD-1-blockade versus IC in MC38-bearing mice on LAG-3 detection in the submandibular lymph nodes. (a) Uptake levels of LAG-3 nanobody tracer in submandibular lymph nodes of anti-PD-1 or IC-mAb treated mice, as determined by ex vivo $\gamma$-counting. (b) Representative axial SPECT/CT-images of submandibular lymph nodes of anti-PD-1 or IC-mAb treated mice i.v. injected with 99mTc-labeled LAG-3, scaled at 0-3 \% IA/ $/ \mathrm{cm}^{3}$ (above) and 0-1.5 \% IA/cm ${ }^{3}$ (below). 